\title{
Virtual lesions of the IFG abolish response facilitation for biological and non-biological cues
}

\author{
Roger D. Newman-Norlund ${ }^{1}$, Sasha Ondobaka ${ }^{2,3}$, Hein T. van Schie ${ }^{4}$, Gijs van Elswijk ${ }^{2,3}$ and Harold Bekkering ${ }^{2,3}$ \\ Department of Exercise Science, University of South Carolina, Columbia, SC, USA \\ 2 Donders Institute for Brain, Cognition and Behavior, Center for Cognition (DCC), Center for Cognitive Neuroimaging (DCCN), \\ Radboud University Nijmegen, Nijmegen, Netherlands \\ 3 Donders Institute for Brain, Cognition and Behavior, Center for Neuroscience, Radboud University Nijmegen, Nijmegen, Netherlands \\ ${ }^{4}$ Behavioural Science Institute, Radboud University Nijmegen, Nijmegen, Netherlands
}

\section{Edited by:}

Dominique J F. de Quervain, University of Basel, Switzerland

Reviewed by:

Elena Rusconi, University College London, UK

Hugo Théoret, Université de Montréal,

Canada

\section{*Correspondence:}

Roger D. Newman-Norlund,

Department of Exercise Science,

University of South Carlina, Columbia,

SC 28208, USA.

e-mail: rnorlund@mailbox.sc.edu
Humans are faster to perform a given action following observation of that same action. Converging evidence suggests that the human mirror neuron system (MNS) plays an important role in this phenomenon. However, the specificity of the neural mechanisms governing this effect remain controversial. Specialist theories of imitation suggest that biological cues are maximally capable of eliciting imitative facilitation. Generalist models, on the other hand, posit a broader role for the MNS in linking visual stimuli with appropriate responses. In the present study, we investigated the validity of these two theoretical approaches by disrupting the left and right inferior frontal gyrus (IFG) during the preparation of congruent (imitative) and incongruent (complementary) actions cued by either biological (hand) or non-biological (static dot) stimuli. Delivery of TMS over IFG abolished imitative response facilitation. Critically, this effect was identical whether actions were cued by biological or non-biological stimuli. This finding argues against theories of imitation in which biological stimuli are treated preferentially and stresses the notion of the IFG as a vital center of general perception-action coupling in the human brain.

Keywords: imitation, ideo-motor, associative sequence learning, perception-action coupling, pars opercularis, transcrania magnetic stimulation

\section{INTRODUCTION}

A well-studied phenomenon in the motor literature is that humans are faster to execute a movement after observing another actor make the same movement even in cases where the observed action is irrelevant to the task (Brass et al., 2000, 2001; Sturmer et al., 2000; Craighero et al., 2002). In the typical experiment of this variety, participants observe a simple finger/hand movement being made by an actor and then respond by either generating the same (congruent) or a different (incongruent) action. Reaction times following presentation of congruent stimuli are normally found to be faster than those following presentation of incongruent stimuli. Results from neuroimaging and virtual lesion experiments suggest that the mirror neuron system (MNS), particularly the inferior frontal gyrus (IFG), plays an important role in perception-action coupling proposed to underlie imitation in general (Iacoboni et al., 1999, 2001; Kilner et al., 2003, 2009; Buccino et al., 2004; Molnar-Szakacs et al., 2005). More recent experiments have linked activity in the IFG to response facilitation (Heiser et al., 2003; Newman-Norlund et al., 2007) as well as stimulus-response compatibility and spatial attention effects which are thought to, in some cases underlie this phenomenon (Dassonville et al., 2001). Despite numerous studies on the topic, the exact neural mechanisms underlying imitative facilitation still remain unclear (Brass et al., 2005; Makuuchi, 2005; Hurley, 2008). In particular, there is some controversy about the exact functional role of the IFG in translating perception into subsequent actions (Jacob and Jeannerod, 2005; Makuuchi, 2005; Hamilton and Grafton, 2008), as well as possible functional differences between its left, right, dorsal and ventral subdivisions.
A number of theories have been put forward to explain the neural mechanisms underlying imitative facilitation. These theories fall into two categories, specialist and generalist (Brass and Heyes, 2005). Specialist theories of imitation, such as the active inter-modal mapping (AIM) theory (Meltzoff and Moore, 1977), propose that a special-purpose imitation system is engaged during the observation of human actions. Data from recent experiments suggests that the IFG does indeed play a specific role in the imitation of biological movements (Heiser et al., 2003). Generalist theories, such as associative sequence learning theory (ASL), assume that imitation is based on general-purpose learning and motor control mechanisms (Heyes, 2001; Brass and Heyes, 2005). While consistent with the generalist perspective, other recent experiments have failed to show preferential activation of the IFG during observation of human actions (Jonas et al., 2007). One way to examine the validity of these two theories is to compare responses to external cues which can evoke motor resonance (e.g. biological stimuli such as hands) to those that cannot (e.g. non-biological stimuli, such as geometric shapes), while disrupting processing in the IFG.

Here, we combined an imitation grasping paradigm with an online 'virtual lesion' approach (Walsh and Rushworth, 1999) to examine the role of the IFG in the facilitation of actions triggered by biological and non-biological cues (Figure 1).TMS pulses delivered over the left and right IFG transiently disrupted the coupling between observed and executed actions. We predicted that stimulation of the IFG would disrupt action planning. Furthermore, we investigated the validity of specialist and generalist based explanations of imitative facilitation by comparing the pattern of responses in biologically 
A

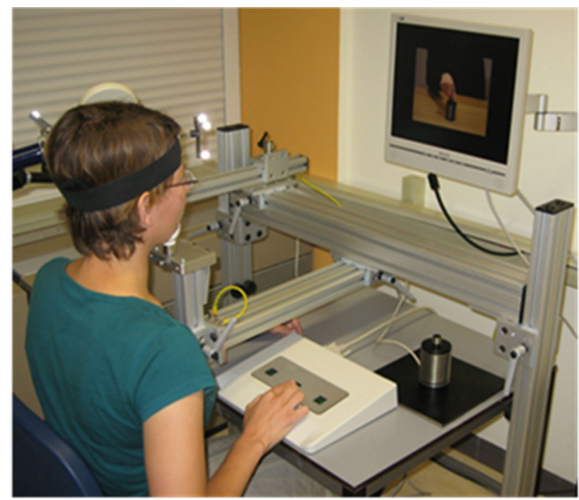

B
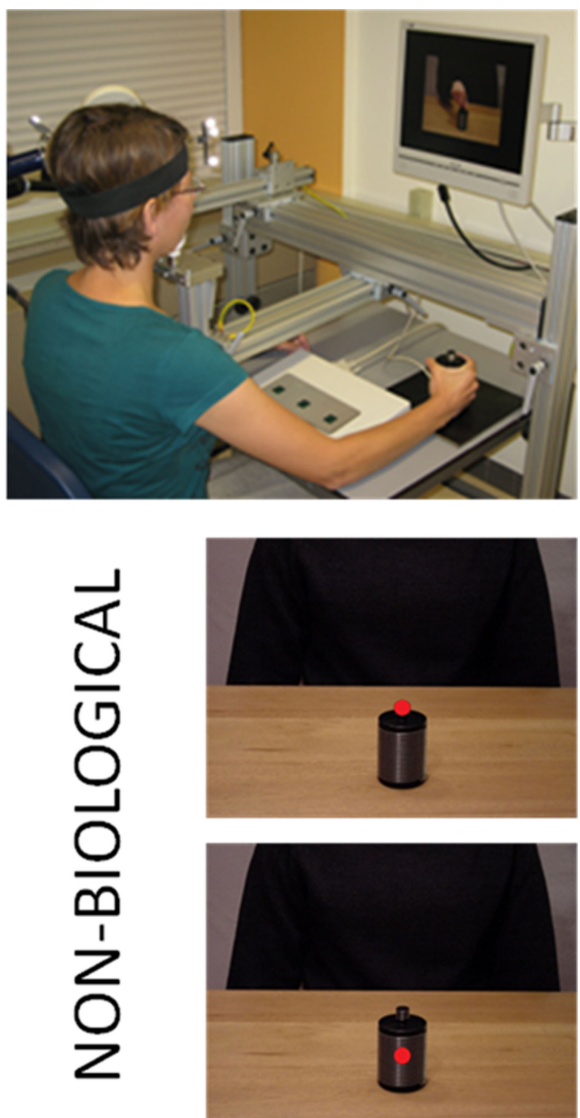

FIGURE 1 | (A) Experimental setup. Participants viewed stimuli presented on a screen directly in front of them. Responses were made using the right hand only. (B) Examples of stimuli used in the biological and non-biological conditions. Biological stimuli consisted of still photographs in which an actor grasped the manipulandum with their right hand using either a precision or a power grip.
Actions in the non-biological condition were cued by the presentation of a red dot on the aspect of the large or small cylinder. In congruent trials, participants copied the grip and gripped the cylinder with the red dot respectively. In incongruent trials, participants performed the opposite grip or gripped the cylinder without a dot respectively. and non-biologically cued trials. While specialist theories predict superior reaction times in response to biological stimuli, generalist theories would be consistent with either (i) superior reaction times to biological stimuli (this might result from associative learning) or (ii) statistically similar reaction times to biological and non-biological stimuli. We show that the delivery of TMS pulses to the IFG during movement preparation disrupts the typical imitative facilitation in both biologically and non-biologically cued conditions.

\section{MATERIALS AND METHODS}

Sixteen right-hand dominant (Oldfield, 1971) healthy adults (19-27 years, mean $=21.5$; six males $)$ with normal or correctedto-normal vision participated in the experiment. Prior to the start of the experiment participants provided their written informed consent and were screened to rule out any history of neurological, psychiatric and medical illness, or contra-indications to TMS. Participants were selected from an initial pool of 27 candidates which had been invited for a pre-test session. During the pre-test we assessed their active motor threshold and their subjective sensitivity to TMS pulses delivered over the IFG. For the actual experiment, we selected the sixteen subjects with the lowest active motor thresh- olds. The purpose of the pre-test was two-fold. First, we ensured that subjects were comfortable with our TMS stimulation protocol before exposing them to extensive testing. Second, we minimized the potentially confounding effect of facial muscle twitches typically observed during IFG stimulation (Pobric and Hamilton, 2006). The protocol was approved by the local ethics committee in accordance with the declaration of Helsinki. Volunteers were compensated for their participation at the rate of $17 €$ per hour.

\section{TESTING PROCEDURE}

Participants were seated at a table facing a computer monitor mounted on a wall bracket at a distance of approximately $70 \mathrm{~cm}$. A chin rest was used to minimize subjects' head movements throughout the experiment. A centrally located response-box, fixed on the table in front of the participant, served as a starting position for their right index finger. In front of the response-box, at a distance of approximately $27 \mathrm{~cm}$, a custom made touch-sensitive manipulandum van Schie et al. (2008), was secured to the table. The manipulandum consisted of a small cylinder $(r=0.8 \mathrm{~cm}$, height $=1.80 \mathrm{~cm})$ on top of a larger cylindrical base $(r=3.00 \mathrm{~cm}$, height $=8.00 \mathrm{~cm})$ that could be grasped in two possible ways. The small and large 
cylinders could be grasped with a precision and power grip, respectively. All stimuli were presented using Presentation software version 11.3 (Neurobehavioral Systems, Davis, CA, USA), running on a Pentium IV PC. Both the response-box and the manipulandum were connected to the PC to detect in each trial: (i) the precise time at which the start button was released following presentation of the imperative stimulus, (ii) the time at which the object was grasped, and (iii) the end-position of the grasp. From this information we were able to calculate reaction time (RT), movement time (MT) and the manner in which the object was grasped (power or precision grip).

Participants performed eight blocks of forty trials each. These blocks constituted a 2 (congruent, incongruent) $\times 4$ (control sites: sham and vertex, IFG sites: right and left) design in which participants performed incongruent and congruent actions while receiving TMS at each of the four stimulation sites. In the congruent task block, participants were instructed to grasp the part of the manipulandum indicated by the target stimulus. In the incongruent task block, they were asked not to grasp the part of the manipulandum indicated by the target stimulus (i.e., to grasp the other part). The order of these blocks was counterbalanced across subjects using a Latin squares design. Every block consisted of twenty trials of each cue type (biological, non-biological).

Each trial started with the participant's right index finger pressing down on the start button of the response-box. First, a fixation cross appeared on the screen which served as a temporal jitter (3000-5000 ms) followed by a $1000 \mathrm{~ms}$ warning stimulus showing the torso and upper arms of an actor in a resting position facing the manipulandum (see Figure 2). Then, the target stimulus appeared and remained on until the computer recorded a grasping response. The target was chosen pseudo-randomly from the two biological and two symbolic stimuli. Biological target stimuli consisted of static pictures of the hand appearing on the manipulandum. Presentation of these stimuli resulted in apparent motion and the perception that the hand moved towards and grasped the manipulandum. The observed grip was either a precision grip or a full grip. Symbolic target stimuli consisted of a red dot which appeared either on the small top cylinder or the larger cylindrical base. In order to prevent on-line switches in their movement, participants were instructed to only initiate the movement when

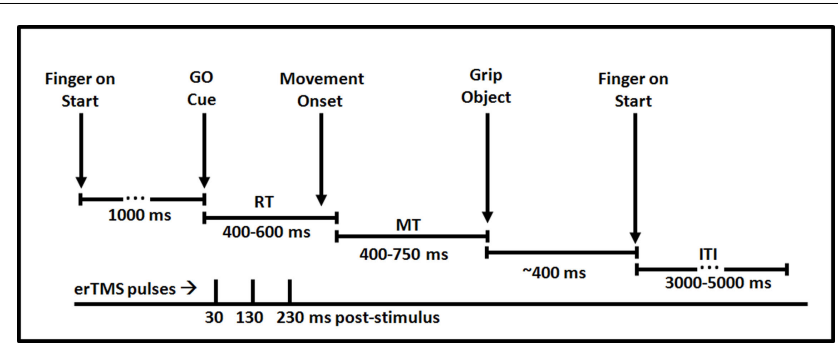

FIGURE 2 |Time course of a single trial in the current experiment. Participants began with their finger on a centrally located start button. Following presentation of a critical go-cue (biological or non-biological), participants prepared and executed the appropriate movement. TMS pulses were delivered 30, 130 and 230 ms post-stimulus. After gripping the manipulandum participants returned their finger to the start button and awaited presentation of the subsequent trial. they were certain that the planned grasp was the correct one. All movements were made with the right hand. Following movement execution, participants returned their hand to the start position for the next trial.

\section{TRANSCRANIAL MAGNETIC STIMULATION PROTOCOL}

The MNI coordinates $(41,15$, and 32$)$ for the right IFG stimulation site were determined using data from a previous fMRI experiment that used the same grasping paradigm as the current experiment (Newman-Norlund et al., 2007). Activation from the statistical parametric map (SPM) based on a random effects analysis of 19 subjects indicated a common reliance of S-R coupling for congruent and incongruent actions on the IFG (Newman-Norlund et al., 2007). This statistical map was reverse normalized to individual subject's high resolution T1 scans obtained prior to the TMS session. The stimulation site for the left IFG site was determined by flipping the coordinates of the right IFG along the $y$ axis. Additionally, we used two TMS control conditions. Sham stimulation was used to control for the clicking sound produced by the TMS pulses. For the sham condition, the coil was placed $4-5 \mathrm{~cm}$ above the primary motor cortex, resulting in the same sound as during IFG stimulation, but without cortical effects of the pulses. To control for any general, non-specific effects of TMS application we chose the vertex site, defined on individual bases as the meeting point of the post-central gyri from the both hemispheres (Ruff et al., 2006).

TMS was delivered using a $70 \mathrm{~mm}$ figure-of-eight coil connected to a Magstim Super Rapid Stimulator (Magstim, Whitland, UK). At the beginning of each block the coil was positioned over one of the four target sites using the subject's T1 scans in combination with a frameless stereotaxy system (Brainsight TMS, Rogue Research; Montreal, Canada). The coil was held tangential to the scalp with its handle pointing backwards and fixed with a mechanical arm. During the experimental blocks the coil position was monitored continuously and adjusted whenever its distance to the target position exceeded $5 \mathrm{~mm}$.

On each trial a triplet of TMS pulses was applied. The pulses were spaced at an interval of $100 \mathrm{~ms}$ and were delivered at 30-130$230 \mathrm{~ms}$ following target stimulus onset. This timing of this pulse train was designed to maximally impair movement preparation. Based on the assumption that the effect of single TMS pulses within a triplet lasts less than $100 \mathrm{~ms}$ (Sack et al., 2005), it is unlikely that the stimulation protocol influenced movement execution. Pulse intensity was set to $100 \%$ of the subject's active motor threshold. The active motor threshold was determined from the first dorsal interosseus muscle while the subject maintained a slight voluntary contraction. Active motor threshold was defined as the lowest level of stimulation that produced at least three motor-evoked potentials of $>200 \mu \mathrm{V}$, out of five consecutive stimulations. The stimulation intensities used during the actual experiment ranged from $37 \%$ to $52 \%$ (average $44.7 \%$ ) of maximum stimulator output $(2.0 \mathrm{~T})$.

\section{RESULTS}

\section{TRIAL EXCLUSION}

From the original 5120 trials from sixteen subjects, 5.8\% were excluded due to erroneous responses (1.6\% start button error, $4.2 \%$ incorrect responses). From the remaining 4822 trials with 
the correct responses, $2.2 \%$ were excluded as outliers $(0.3 \%$ due to reaction times and MTs less than $100 \mathrm{~ms}$ and greater than $1200 \mathrm{~ms} ; 1.9 \%$ due to reaction times and MTs 2.5 SD cutoff for each subject for each individual block). The remaining correct trials were subjected to a three way $(2 \times 2 \times 2)$ repeatedmeasures ANOVA on mean reaction times and mean movement times. Because the effects of sham and vertex stimulation were statistically indistinguishable, these conditions were collapsed into a single 'control' condition. We used cue type (biological, non-biological), TMS stimulation (IFG, control) and action congruency (congruent, incongruent) as within subjects' factors. Analysis of MTs results did not yield any significant interactions and showed only a significant main effect of cue type $\left(F_{(1,15)}=4.78 ; P<0.05\right)$ indicating shorter MTs for biologically cued movements $($ mean $=504, \mathrm{SD}=97)$ as compared to nonbiologically cued movements (mean $=510, \mathrm{SD}=94)$. In the following we focus mainly the RT results and do not discuss MT results in greater detail due to our predictions being on the level of response times and the fact that we found no significant interaction effects in the MT results.

\section{REACTION TIMES ANOVA}

The main effect of cue type was significant $\left(F_{(1,15)}=22.80 ; P<0.001\right)$, indicating faster reaction times for non-biological ( mean $=468$, $\mathrm{SD}=61)$ as compared to biological targets $($ mean $=484, \mathrm{SD}=60)$. The main effect of action congruency also showed a significant effect $\left(F_{(1,15)}=5.39 ; P<0.05\right)$, with participants showing an overall faster reaction as compared to the congruent trials (mean $=470$, $\mathrm{SD}=66$ ) than in the incongruent trials (mean $=483, \mathrm{SD}=57$ ). The main effect of TMS stimulation failed to reach significance $\left(F_{(1,15)}=2.96 ; P=0.11\right)$.

Our exploration of two-way interactions revealed a significant interaction effect between TMS stimulation and action congruency $\left(F_{(1,15)}=6.81 ; P<0.05\right)$. Specifically, this indicated that TMS stimulation of IFG abolished the RT facilitation effect observed in congruent trials with control TMS stimulation (Figure 3, Figure S1 in Supplementary Material). We found no interaction between TMS stimulation and cue type $\left(F_{(1,15)}=0.14 ; P=0.72\right)$, indicating no differential effect of TMS on condition (biological, non-biological). Furthermore, we found no significant interaction between cue type and congruency $\left(F_{(1,15)}<1 ; P=0.97\right)$ demonstrating that biological and non-biological cues did not differentially influence the pattern of reaction times observed in the congruent and incongruent conditions.

In order to further specify the effects of TMS on action congruency we ran post hoc t-tests comparing reaction times in the congruent and incongruent trials separately for the biological and non-biological conditions. Analysis of biologically cued trials revealed a significant facilitation of responses by $28 \pm 7.4 \mathrm{~ms}$ (mean \pm SEM; $t_{15}=-3.75 ; P<0.005$ ) for congruent trials relative to incongruent trials during control TMS. Similarly, the results from the non-biological condition also revealed a facilitation effect of $25 \pm 6.0 \mathrm{~ms}$ (mean $\pm \mathrm{SEM} ; t_{15}=-4.18 ; P<0.005$ ) for congruent trials as compared to the incongruent trials during control TMS. Crucially, this reaction time advantage disappeared during stimulation of the IFG in both the biological $\left(t_{15}<1 ; P=0.80\right)$ and non-biological conditions $\left(t_{15}<1 ; P=0.97\right.$; Figure 3).

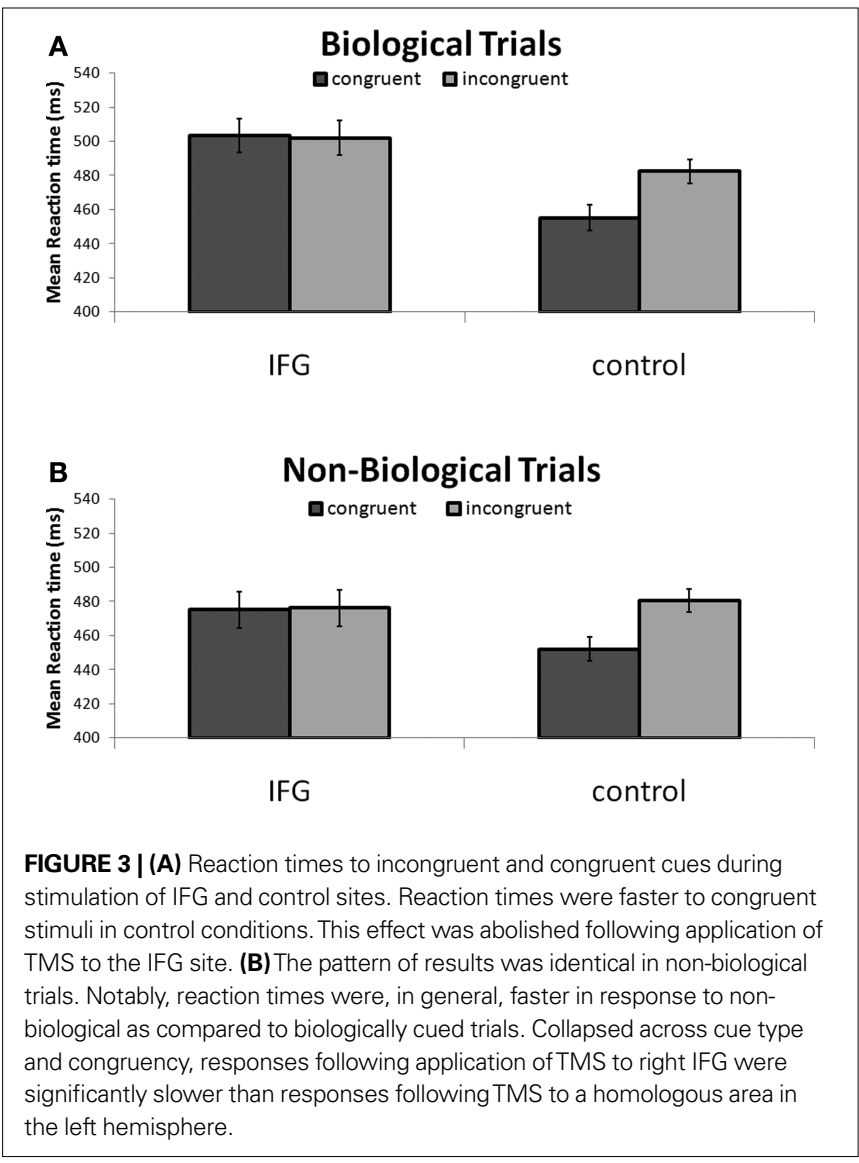

\section{DISCUSSION \\ SPECIALIST VERSUS GENERALIST THEORIES OF PERCEPTION-ACTION COUPLING}

The major goal of the current experiment was to examine the neural and functional mechanisms underlying imitative response facilitation. Here, we explicitly tested the validity of two classes of theories, specialist and generalist, which have been proposed to explain this phenomenon. Consistent with the experiments indicating a critical role of the left and right IFG in perception-action coupling (Iacoboni et al., 2001; Buccino et al., 2004; Aziz-Zadeh et al., 2006; Newman-Norlund et al., 2007) triple-pulse TMS over both the left and right IFG sites abolished the imitative facilitation effect. Critically, we demonstrate that the IFG is equally involved the preparation of responses following observation of both biological and non-biological stimuli. This was evident from the same general pattern of reaction times we observed in biologically and non-biologically cued trials. The fact that the processing of biological and non-biological stimuli was not differentially effected by disruption of IFG argues strongly against specialist theories of imitation and is more compatible with theories positing a more flexible role of MNS in perception-action coupling required for the preparation of movements cued by external stimuli.

One previous experiment using on-line repetitive TMS to disturb processing in the IFG during imitation found a differential effect of TMS stimulation on error rates for biologically and non-biologically cued movements (Heiser et al., 2003). Specifically, they 
found a greater error rate for biological trials following both left and right TMS in comparison to control TMS trials. This finding is entirely consistent with our reaction time results for biological trials indicating that normal IFG activity is crucial for perception-action coupling during imitation of biological actions. In contrast to our results, Heiser and colleagues found no differential effect of rTMS stimulation of the IFG on error rates in a non-biological condition. This led the authors to conclude that disruption of the IFG interfered with a mechanism specifically involved in imitation. However, it has been suggested that in explaining results of imitation paradigms the biological properties of a stimulus may be less important than task specific requirements which are embedded in the experimental context (Pobric and Hamilton, 2006; Jonas et al., 2007). Consistent with this proposal, discrepancies between the current and previous findings could be explained by methodological differences between the two experiments. In their biological condition (imitation), Heiser et al. (2003) presented short video clips of two sequential finger presses which had to be imitated. In the non-biological condition (control) the participants had to respond to a red dot which would appear over a sequence of two keys. Here, the position of the dot was always spatially compatible with the moved finger. In the imitation condition, participants were instructed to make their first key press using the same finger used by the actor. In these trials, the location of the participants' finger and the spatial location of the key being pressed on the keyboard were not always spatially compatible. Arguably, this confound in the spatial congruency between the two conditions could account for the differential reliance on IFG processing found in imitation and control tasks. Furthermore, differences between the present and previous findings from Heiser et al. (2003) could be due to the exact location of TMS delivery within the IFG. We targeted the dorsal portion of pars opercularis which is thought to be the best candidate for translating visual to motor information, since it has been found to be active during both execution and observation of imitative actions (Molnar-Szakacs et al., 2005). In comparison, Heiser et al. (2003) targeted a more ventral portion of pars opercularis which, in the same meta-analysis, did not exhibit mirror-like responses. Future experiments might evaluate the roles of different portions of pars opercularis by delivering TMS at multiple sites along the dorsal-ventral axis of pars opercularis during similar tasks.

While TMS clearly had an effect on reaction times to congruent stimuli, reaction times to incongruent stimuli in the TMS conditions were not different from reaction times to incongruent stimuli in the control conditions. This is similar to results from Heiser and colleagues who found that TMS pulses delivered over left and right IFG impaired performance on an imitation task, but not on a comparable control task (Heiser et al., 2003). These results strongly imply that disruption of the IFG selectively impairs a neural mechanism that is highly specific to imitation. The fact that incongruent trials were unaffected by TMS in the present experiment suggests that this imitation mechanism was not required for their successful completion. This result may also be related to higher cognitive demands in the incongruent condition (reflected in the RTs difference between the two conditions). Specifically, it may be the case that the top-down control processes engaged to overcome interference arising during incongruent trials are responsible for the lack of effect (Badre and Wagner, 2006; Brass et al., 2009).

\section{BIOLOGICALLY VERSUS NON-BIOLOGICALLY CUED RESPONSES}

In the current experiment, reaction times were faster for nonbiologically as compared to biologically cued stimuli. Previous experiments in which movement execution was cued using biological and non-biological cues found the opposite effect, with actions cued by natural stimuli eliciting significantly faster reaction times (Brass et al., 2000; Jonas et al., 2007). The inconsistency between our results and prior behavioral results is mirrored in the conflicting results of recent neuroimaging investigations comparing neural responses to biological and non-biological stimuli. Some data support the idea that biological movements bias the motor system in a fundamentally different way than non-biological motions (Iacoboni et al., 1999; Stevens et al., 2000; Perani et al., 2001; Heiser et al., 2003; Kilner et al., 2003; Tai et al., 2004; Kessler et al., 2006). Other data, however, find that observation of biological and nonbiological stimuli (e.g. robot actions) activates largely overlapping neural structures (Gazzola et al., 2007) with one experiment finding no differences in mirror areas when imitation was prompted by biological and non-biological cues (Jonas et al., 2007). Consistent with the latter view, a recent study by Jansson et al. (2007) suggests that many of the behavioral differences originally attributed to preferential processing of biological stimuli can be explained by physical differences between stimuli, including but not limited to differences in saliency and the degree of congruence between stimuli and response. In their study, when biological and symbolic action cues were carefully matched in the visual dimension, comparable cueing effects were found for both conditions (Jansson et al., 2007). Consistent with this line of reasoning, the red dots used in the nonbiological condition in the present experiment may have been more salient than the hands in the biological condition.

\section{INTERPRETATIONAL CAVEATS}

One obvious difference between the present study and previous empirical investigations of imitative facilitation, is that, here, we used a randomized design in which biological and non-biological cues were intermixed within a single experimental block. Previous experiments typically had both types of cueing in separate blocks. One possibility is that the strategy of selectively attending to spatial aspects of the symbolic cues influenced the distribution of spatial attention in the biological condition. That is, in the context of the present task hand-cues may have been be less efficient than dot stimuli in directing attention to the location for grasping (e.g. because hands are less spatially confined). We cannot rule out the possibility that, had these conditions been run in separate blocks, alternative strategies may have developed.

A second possible issue regarding our interpretation of the current data involves the nature of the facilitation effects we report. Previous experiments have distinguished between facilitation effects related to spatial congruency and movement congruency (Brass et al., 2000; Press and Heyes, 2008; van Schie et al., 2008). The primary concern here is that the facilitation effects observed in the current experiment may simply reflect the spatial congruency between stimulus and response, and thus not necessarily be related to movement imitation per se. This could explain why the results were similar in the biological and non-biological conditions. Although one previous behavioral experiment using a similar gripping paradigm (van Schie et al., 2008) found evidence 
consistent with the presence of both spatial and movement based compatibility effects, the current experiment did not include the necessary conditions to make the same comparison. Indeed, it is difficult to disentangle the neural representations of these congruency effects. One possibility, consistent with a recent proposal by Press et al. (2005), is that both types of compatibility effects result from the same associative learning process, a process in which, according to the present findings, the IFG appears to play a critical role.

\section{SUMMARY}

In the present experiment we investigated the validity of two general classes of theories, specialist and generalist, capable of explaining imitative facilitation using a grasping paradigm in which participants produced congruent or incongruent actions in response to biological or non-biological cues. Facilitation of congruent actions was abolished by 'virtual lesions' of the IFG. Critically, this pattern of disruption was identical whether actions were triggered by biological or non-biological cues. This finding argues against theoretical models of imitation in which the IFG is hypothesized to play a special role in the processing of

\section{REFERENCES}

Aziz-Zadeh, L., Koski, L., Zaidel, E., Mazziotta, J., and Iacoboni, M. (2006). Lateralization of the human mirror neuron system. J. Neurosci. 26, 2964-2970.

Badre, D., and Wagner, A. D. (2006). Cognitive and neurobiological mechanisms underlying cognitive flexibility. Proc. Natl. Acad. Sci. USA 103, 7186-7191.

Brass, M., Bekkering, H., and Prinz, W. (2001). Movement observation affects movement execution in a simple response task. Acta Psychol. (Amst) 106, 3-22.

Brass, M., Bekkering, H., Wohlschlager,A., and Prinz, W. (2000). Compatibility between observed and executed finger movements: comparing symbolic, spatial, and imitative cues. Brain Cogn. 44, 124-143.

Brass, M., Derrfuss, J., and von Cramon, D. Y. (2005). The inhibition of imitative and overlearned responses: a functional double dissociation. Neuropsychologia 43, 89-98.

Brass, M., and Heyes, C. (2005). Imitation: is cognitive neuroscience solving the correspondence problem? Trends Cogn. Sci. 9, 489-495.

Brass, M., Wenke, D., Spengler, S., and Waszak, F. (2009). Neural correlates of overcoming interference from instructed and implemented stimulusresponse associations. J. Neurosci. 29, 1766-1772.

Buccino, G., Vogt, S., Ritzl, A., Fink, G. R., Zilles, K., Freund, H. J., and Rizzolatti, G. (2004). Neural circuits underlying imitation learning of hand actions: an event-related fMRI study. Neuron 42, 323-334.

Craighero, L., Bello, A., Fadiga, L., and Rizzolatti, G. (2002). Hand action preparation influences the responses to hand pictures. Neuropsychologia 40, 492-502.

Dassonville, P., Lewis, S. M., Zhu, X. H., Ugurbil, K., Kim, S. G., and Ashe, J. (2001). The effect of stimulus-response compatibility on cortical motor activation. Neuroimage 13, 1-14.

Gazzola, V., Rizzolatti, G., Wicker, B., and Keysers, C. (2007). The anthropomorphic brain: the mirror neuron system responds to human and robotic actions. Neuroimage 35, 1674-1684.

Hamilton, A. F., and Grafton, S. T. (2008). Action outcomes are represented in human inferior frontoparietal cortex. Cereb. Cortex 18, 1160-1168.

Heiser, M., Iacoboni, M., Maeda, F., Marcus, J., and Mazziotta, J. C. (2003). The essential role of Broca's area in imitation. Eur. J. Neurosci. 17, 1123-1128.

Heyes, C. (2001). Causes and consequences of imitation. Trends Cogn. Sci. (Regul. Ed.) 5, 253-261.

Hurley, S. (2008). The shared circuits model (SCM): how control, mirroring, and simulation can enable imitation, deliberation, and mindreading. Behav. Brain Sci. 31, 1-22; discussion 22-58.

Iacoboni, M., Koski, L. M., Brass, M., Bekkering, H., Woods, R. P., Dubeau, M. C., Mazziotta, J. C., and Rizzolatti, G. (2001). Reafferent copies of imitated actions in the right superior

human stimuli. It is more consistent with the proposal that the IFG subserves the more general purpose of perception-action coupling (Liepelt et al., 2008). Overall, the present findings stress the notion that the IFG supports a general perception-action coupling mechanism necessary for linking our own actions to events perceived in the environment, whether they are biological actions or not.

\section{ACKNOWLEDGMENTS}

The present study was supported by the EU-Project 'Joint-Action Science and Technology' (IST-FP6-003747). We would additionally like to thank Sebastiaan Overeem, Dick Stegeman, Ivan Toni, Sarah Newman-Norlund, Matthijs Noordzij, Pascal de Water, Norbert Hermesdorf, Erik Hogervorst and Paul Gaalman for their valuable assistance.

\section{SUPPLEMENTARY MATERIAL}

The Supplementary Material for this article can be found online at http://www.frontiersin.org/behavioralneuroscience/paper/10.3389/ neuro.08/005.2010/

temporal cortex. Proc. Natl. Acad. Sci. USA 98, 13995-13999.

Iacoboni, M., Woods, R. P., Brass, M. Bekkering, H., Mazziotta, J. C., and Rizzolatti, G. (1999). Cortical mechanisms of human imitation. Science 286, 2526-2528.

Jacob, P., and Jeannerod, M. (2005). The motor theory of social cognition: a critique. Trends Cogn. Sci. (Regul. Ed.) 9, 21-25.

Jansson, E., Wilson, A. D., Williams, J. H., and Mon-Williams, M. (2007). Methodological problems undermine tests of the ideo-motor conjecture. Exp. Brain Res. 182, 549-558.

Jonas, M.,Siebner,H.R.,Biermann-Ruben, K., Kessler, K., Baumer, T., Buchel, C., Schnitzler, A., and Munchau, A. (2007). Do simple intransitive finger movements consistently activate frontoparietal mirror neuron areas in humans? Neuroimage 36(Suppl. 2), T44-T53.

Kessler, K., Biermann-Ruben, K., Jonas, M., Siebner, H. R., Baumer, T., Munchau, A., and Schnitzler, A. (2006). Investigating the human mirror neuron system by means of cortical synchronization during the imitation of biological movements. Neuroimage, 33, 227-238.

Kilner, J. M., Neal, A., Weiskopf, N. Friston, K. J., and Frith, C. D. (2009). Evidence of mirror neurons in human inferior frontal gyrus. J. Neurosci. 29, 10153-10159.

Kilner, J.M., Paulignan, Y., and Blakemore, S. J. (2003). An interference effect of observed biological movement on action. Curr. Biol. 13, 522-525.
Liepelt, R., Cramon, D. Y., and Brass, M (2008). What is matched in direct matching? Intention attribution modulates motor priming. J. Exp. Psychol. Hum. Percept. Perform. 34, 578-591.

Makuuchi, M. (2005). Is Broca's area crucial for imitation? Cereb. Cortex 15, 563-570.

Meltzoff, A. N., and Moore, M. K. (1977). Imitation of facial and manual gestures by human neonates. Science 198, 74-78.

Molnar-Szakacs, I., Iacoboni, M., Koski, L., and Mazziotta, J.C. (2005). Functional segregation within pars opercularis of the inferior frontal gyrus: evidence from fMRI studies of imitation and action observation. Cereb. Cortex 15, 986-994.

Newman-Norlund, R. D., van Schie, H. T., van Zuijlen, A. M., and Bekkering, H. (2007). The mirror neuron system is more active during complementary compared with imitative action. Nat. Neurosci. 10 817-818.

Oldfield, R.C. (1971). The assessment and analysis of handedness: the Edinburgh inventory. Neuropsychologia 9, 97-113.

Perani, D., Fazio, F., Borghese, N. A., Tettamanti, M., Ferrari, S., Decety, J., and Gilardi, M. C. (2001). Different brain correlates for watching real and virtual hand actions. Neuroimage 14, 749-758.

Pobric, G., and Hamilton, A. F. (2006). Action understanding requires the left inferior frontal cortex. Curr. Biol. $16,524-529$. 
Press, C., Bird, G., Flach, R., and Heyes, C. (2005). Robotic movement elicits automatic imitation. Brain Res. 25, 632-640.

Press, C., and Heyes, C. (2008). Stimulusdriven selection of routes to imitation. Exp. Brain Res. 188, 147-152.

Ruff, C. C., Blankenburg, F., Bjoertomt, O., Bestmann, S., Freeman, E., Haynes, J. D., Rees, G., Josephs, O., Deichmann, R., and Driver, J. (2006). Concurrent TMSfMRI and psychophysics reveal frontal influences on human retinotopic visual cortex. Curr. Biol. 16, 1479-1488.

Sack, A. T., Camprodon, J. A., PascualLeone, A., and Goebel, R. (2005). The dynamics of interhemispheric compensatory processes in mental imagery. Science 308, 702-704.

Stevens, J. A., Fonlupt, P., Shiffrar, M., and Decety, J. (2000). New aspects of motion perception: selective neural encoding of apparent human movements. Neuroreport 11, 109-115.

Sturmer, B., Aschersleben, G., and Prinz, W. (2000). Correspondence effects with manual gestures and postures: a study of imitation. J. Exp. Psychol. Hum. Percept. Perform. 26, 1746-1759.

Tai, Y. F., Scherfler, C., Brooks, D. J., Sawamoto, N., and Castiello, U. (2004). The human premotor cortex is 'mirror' only for biological actions. Curr. Biol. 14, 117-120. van Schie, H. T., van Watershoot, B.M., and Bekkering, H. (2008). Understanding action beyond imitation: reversed compatibility effects of action observation in imitation and joint action. J. Exp. Psychol. Hum. Percept. Perform. 34, 1493-1500.

Walsh, V., and Rushworth, M. (1999). A primer of magnetic stimulation as a tool for neuropsychology. Neuropsychologia 37, 125-135.

Conflict of Interest Statement: The authors declare that the research was conducted in the absence of any commercial or financial relationships that could be construed as a potential conflict of interest.
Received: 04 September 2009; paper pending published: 25 November 2009; accepted: 02 February 2010; published online: 03 March 2010.

Citation:Newman-Norlund RD, Ondobaka S, van Schie HT, van Elswijk GandBekkering H. (2010) Front. Behav. Neurosci. 4:5. doi: 10.3389/neuro.08.005.2010

Copyright (c) 2010 Newman-Norlund, Ondobaka, van Schie, van Elswijk and Bekkering. This is an open-access article subject to an exclusive license agreement between the authors and the Frontiers Research Foundation, which permits unrestricted use, distribution, and reproduction in any medium, provided the original authors and source are credited. 